\title{
Migraine Prevalence, Treatment and Impact: The Canadian Women and Migraine Study
}

\author{
Lara J. Cooke, Werner J. Becker
}

\begin{abstract}
Background: The prevalence of migraine headache varies somewhat across geographic regions. The last Canadian population-based study of migraine was in 1994. We report the findings of the Canadian Women and Migraine Survey. In addition to reporting migraine prevalence in Canadian women, the survey identified current consultation and treatment practices of women with migraine, and the psychological burden of migraine. Methods: The survey was conducted with a population-based sample of 1210 women using standard telephone research methods. Headache diagnoses were based on the International Headache Society (IHS) Classification. Results: Calculated prevalence of migraine headache was $26 \%$. Only $51 \%$ of women with migraine had consulted a physician about their headaches. Women with migraines rely on over-the-counter medications and non-specific prescription medications. Less than $10 \%$ of women with migraine use triptans/dihydroergotamine for primary treatment. Ninety seven percent of women with migraine reported at least one psychosocial impact resulting from migraines. Conclusions: The prevalence of migraine in Canadian women appears static, and is again shown to be slightly higher than that reported in the United States. As in other epidemiologic studies, many women with migraine do not seek medical help for their headaches and perhaps as a result, few are using migraine-specific medications to treat their headaches. The impact of migraine on Canadian women is substantial with almost all women with migraine reporting adverse psychosocial effects of migraines on their lives.
\end{abstract}

RÉSUMÉ: Prévalence de la migraine, traitement et impact : The Canadian Women and Migraine Study. Contexte : La prévalence de la migraine varie selon les régions. La dernière étude canadienne de population sur la migraine date de 1994. Nous rapportons les observations de la Canadian Women and Migraine Survey. Cette enquête a identifié entre autres les habitudes de consultation et de traitement actuels des femmes atteintes de migraine et a évalué le fardeau psychologique de la migraine. Méthodes : Cette enquête a été effectuée chez un échantillon de 1210 femmes provenant de la population générale, au moyen de méthodes standards de recherche téléphonique. Les diagnostics de céphalée étaient basés sur la classification de la Société internationale de la céphalée. Résultats : La prévalence de la migraine était de $26 \%$. Seulement $51 \%$ des femmes atteintes de migraine avaient consulté un médecin au sujet de leurs céphalées. Les femmes atteintes de migraine ont recours à des médicaments sans ordonnance et à des médicaments sous prescription qui ne sont pas spécifiques de la migraine. Moins de $10 \%$ des femmes atteintes de migraine utilisaient des triptans/de la dihydroergotamine comme traitement primaire. Quatre-vingt-dix-sept pour cent des femmes atteintes de migraine ont rapporté au moins un impact psychosocial de leurs migraines. Conclusions : La prévalence de la migraine chez les femmes canadiennes semble stable et nous démontrons encore une fois qu'elle est légèrement plus élevée que celle rapportée aux États-Unis. Plusieurs femmes atteintes de migraine ne consultent pas un médecin pour leurs céphalées, comme on l'a d'ailleurs observé dans d'autres études épidémiologiques, ce qui explique sans doute que peu utilisent des médicaments spécifiques de la migraine pour traiter leurs céphalées. L'impact de la migraine chez les femmes canadiennes est substantiel, presque toutes les femmes atteintes de migraine ayant rapporté des effets psychosociaux négatifs des migraines dans leur vie.

Can. J. Neurol. Sci. 2010; 37: 580-587

It is well known that migraine headache is a common condition, affecting women more than men; however local prevalence is important when considering the burden of a condition on a society. No population-based study of migraine in Canada has been published since $1994^{1}$.

O'Brien, Goeree, and Streiner (1994) reported migraine prevalence among Canadian males as $7.8 \%$ and $24.9 \%$ among Canadian females ${ }^{1}$. In 1994, this was estimated to constitute 2.6 million adult women and 0.8 million adult men in Canada. Importantly, the authors also reported that only $46 \%$ of those respondents who met the International Headache Society Criteria for migraine had ever received the diagnosis from their physician $^{1}$. At a time when multiple, specific migraine medications are available, under-diagnosis of this potentially treatable condition could contribute to its substantial burden on our society.

From the Department of Clinical Neurosciences, University of Calgary, Calgary, Alberta, Canada.

Received September 30, 2009. Final Revisions Submitted March 2, 2010. Correspondence to: Lara J. Cooke, Department of Clinical Neurosciences, 12th Floor, Foothills Medical Center, 1403-29th St NW, Calgary, Alberta, T2N 2T9, Canada. 
In this paper, we describe the findings of the Canadian Women and Migraine Study, a population-based telephone survey conducted to determine what proportion of Canadian women suffer from migraine headache, the physical, social, and psychological impact of migraine in this population, and the patterns of use of anti-migraine medications by Canadian women with migraine headaches.

\section{METHODS}

A standardized, population-based national telephone survey (Appendix A) was carried out in Canada for women over age 18 on behalf of Headache Network Canada, a non-profit organization dedicated to providing headache-related education to migraine sufferers and the public. The survey instrument was designed by Merck Frosst Canada Inc. in collaboration with physician members of Headache Network Canada. The survey was conducted by an independent research organization (Baromètre) between July 6th and 24th, 2005.

One-thousand two-hundred and ten subjects were selected at random in a representative distribution across Canada using standard telephone survey protocols. Inclusion criteria were female gender, and being at least 18 years-of-age. In order to avoid the bias of selecting female household members interested in headache, surveyors asked to speak to the female household member whose birthday was most recent. As the study was conducted by an independent firm, at the request of Headache Network Canada, ethics review was not conducted prior to the survey. However, after analyzing the pooled data, the project was

Table 1: Healthcare providers consulted by women with migraine headaches *

\begin{tabular}{ll}
\hline Health Care Practitioner & $\% * *$ \\
\hline Doctor & 83 \\
Neurologist & 15 \\
Nurse & 0 \\
Pharmacist & 1 \\
Ophthalmologist & 1 \\
Osteopath & 1 \\
Homeopath & 0 \\
Acupuncturist & 1 \\
Chiropractor & 6 \\
Massage therapist & 2 \\
Naturopath & 1 \\
Other & 7 \\
\hline
\end{tabular}

* Based on the 188 women in the survey who had consulted a health care professional; ** The percentages add up to more than $100 \%$ because some women consulted more than one health care professional. submitted to the Conjoint Health Research Ethics Board at the University of Calgary, and the committee chair provided a letter to support the work, indicating that it would have received full approval.

The questionnaire was based on the diagnostic criteria for migraine headache from the International Headache Society's Classification of Headache Disorders, 1st Edition, and the Migraine-ACT questionnaire ${ }^{2,3}$. Respondents were asked about their headache features, healthcare resource utilization, the psychosocial burden of migraine and patterns of medication use to treat their headache.

Descriptive statistics, including means and standard errors were calculated for pooled survey responses. Responses to questions about the psychosocial burden of disease were based on multiple choice answers provided to participants, and as such, were tabulated in the same manner as the responses to questions about headache frequency and characteristics. Answers to unstructured questions were recorded verbatim and grouped according to common themes.

\section{RESULTS}

\section{Headache Frequency}

Two-thousand eight-hundred and ninety-five women were contacted using phone numbers selected randomly across the country. The mean sampling error for the entire survey was $2.9 \%$ 19 times out of 20. Female household members were selected randomly by having the interviewer ask to speak to the woman in the home who most recently had a birthday. One thousand two hundred and ten women participated in the telephone survey, a response rate of $41.8 \%$.

Eighty-one percent of women reported suffering from some headaches. When women were asked to rate their headache frequency as often, occasionally, rarely or never, $18 \%$ indicated that "often" best described their headache frequency. Twenty-six percent $(n=300$, CI $23.5 \%$ to $28.5 \%)$ of all respondents reported headache symptoms meeting the criteria for the diagnosis of migraine headache. This group represents $31.8 \%$ of the total group of women who reported suffering from headaches.

\section{Resource Utilization}

Of the 300 women who reported headaches in keeping with a diagnosis of migraine headache, $62 \%$ had consulted any form of healthcare practitioner, and $51.5 \%$ reported consulting a physician about their headaches. Fifteen percent of these individuals had seen a neurologist about their headaches. Of women with frequent migraines, $83 \%$ consulted a health care professional, whereas $39 \%$ of women with rare migraine attacks consulted a health care professional about them.

Thirty-two percent of women between the ages of 18 and 24 consulted health care professionals, while $72 \%$ of women over the age of 45 consulted a health care professional about their migraines. While younger women were less likely to consult a health care professional about migraine headaches, all participants between 18 and 24 who did consult a health care professional consulted a physician. Table 1 shows the healthcare providers consulted about headaches by women with migraine. 
Table 2: Medications used by women with migraine for primary treatment of their headaches

\begin{tabular}{ll}
\hline Medication & Percent for whom it was the primary medication \\
\hline Migraine Drugs & 3 \\
Sumatriptan & 2 \\
Rizatriptan & 1 \\
Almotriptan & 1 \\
Naratriptan & 1 \\
Zolmitriptan & 0 \\
Eletriptan & 1 \\
dihydroergotamine mesylate nasal spray & \\
\hline & \\
\hline Other Prescription Drugs & 18 \\
\hline Acetaminophen -codeine-caffeine & 3 \\
ASA-caffeine-codeine-butalbital & 1 \\
naproxen sodium & $<1$ \\
Oxycodone & 4 \\
other prescriptions & $<1$ \\
Celecoxib & \\
\hline & \\
\hline Over-the-Counter Medications & 38 \\
\hline Ibuprofen & 8 \\
Acetaminophen & 2 \\
other over-the-counter medications & 1 \\
acetylsalicylic acid & 2 \\
\hline & 1 \\
\hline Other Medications & 10 \\
\hline did not know & \\
Nothing & \\
\hline ASA & \\
\hline
\end{tabular}

ASA=acetylsalicylic acid

\section{Patterns of Medication Use}

Ten percent of migraine sufferers reported using no medication for their headaches. With regard to the migraine specific medications, $8 \%$ were using triptans as their principal migraine medication, and $1 \%$ were using dihydroergotamine nasal spray. Twenty three percent were taking codeinecontaining analgesics, including three percent who were taking medications containing codeine and butalbital. Very few patients were taking prescription NSAIDs, but the non-prescription medication use was dominated by ibuprofen, which was used by $38 \%$ of patients. This greatly exceeded the use of acetaminophen alone, used by only $8 \%$ as their main migraine medication (Table 2).

\section{Response to Medication}

Twenty-five percent of women reported that they were still unable to function normally two hours after taking their medication when they have a headache. Sixty-two percent reported that they were likely to be headache-free two hours after taking their medication. Twenty percent reported that they did not find that their medications could be relied upon to work consistently. Further, $19 \%$ indicated that they were not comfortable enough with the effectiveness of their medication to allow them to plan daily activities.
When asked about satisfaction with the medication they used for their headaches, $14 \%$ of women with migraine were either a little unsatisfied or very unsatisfied (Table 3).

When women who were not wholly satisfied with their primary headache medication were asked whether they had ever asked a physician whether there were more effective options, only $38 \%$ responded that they had. Indeed, $16 \%$ indicated that they "don't see doctors for headaches".

\section{Headache Impact}

Headache impact was measured by response to the following question (Item 8a, Appendix A): "How many days in the last six months would you estimate your (migraines / headaches) incapacitated you, including missed work days, difficulty doing housework, and/or caring for children?"

Of the 300 women who had headache meeting migraine diagnostic criteria, 30\% reported being incapacitated for more than 5 days in the past six months, $46 \%$ reported 1 to 5 days, and $20 \%$ reported 0 days. The mean number of missed days per year was 20.8. Those with frequent migraines lost an average of 18.3 days in the previous six months to either reduced function or being totally incapacitated. Those who were not wholly satisfied with their medication lost 11.8 days in the previous six months.

When participants were asked how they respond to the onset of a migraine attack, $65 \%$ reported that they would take 
Table 3: Satisfaction with medication used as primary migraine treatment

\begin{tabular}{ll} 
Response & \% of women with migrainous headaches \\
\hline Very satisfied & 37 \\
Somewhat satisfied & 46 \\
A little unsatisfied & 9 \\
Very unsatisfied & 5 \\
\hline
\end{tabular}

medication, $61 \%$ reported that they would stay in bed until it is over, $19 \%$ indicated they would stay in a dark room for a period of time, and $3 \%$ reported that they would continue with their usual activities.

When asked about the psycho-social burden of migraines, $73 \%$ of participants indicated that they sometimes feel a lack of control over their lives, $92 \%$ missed days at work or family activities, and $75 \%$ experienced a lack of understanding or cynicism from those around them. Participants were more likely to experience a lack of understanding on the part of those around them if they were also not wholly satisfied with their medication $(12 \%$ vs. $3 \%, p<0.05)$. Ninety seven percent of migraine sufferers reported experiencing at least one of the psychosocial burdens listed above.

\section{Discussion}

This study contributes several important findings to the current literature on migraine prevalence and burden. First, we present an update on the prevalence rate for migraine in Canadian women. Further, the data suggest that although the burden of migraine headaches upon Canadian women is very high, the use of migraine-specific treatment and physician consultation about migraine headaches is surprisingly low. Further, we present data on the psychosocial burden of migraine in Canadian women, and show that the societal impact of the condition, measured by days of partial or complete disability is enormous.

\section{Migraine Prevalence in Canada}

In review of previous epidemiological data, we find two large studies of migraine in Canada. In 1992 Pryse-Phillips et al reported a lifetime prevalence of migraine in women of $23 \%{ }^{4}$. O'Brien et al (1994) reported the one-year migraine prevalence among Canadian women to be $24.9 \%{ }^{1}$. These figures are not dissimilar to the six-month prevalence rate reported in this study, of $26 \%$.

In comparison to reported prevalence rates elsewhere in the literature, it appears that the prevalence of migraine in Canadian women is slightly higher than in women in the United States, Germany, France, and England. Reported prevalence rates in the United States vary from $17 \%$ to $18.3 \%{ }^{5-7}$. In England, the one- year prevalence of women with migraine was reported as $18.3 \%^{8}$. In Germany, migraine prevalence in women was reported as $15.6 \%{ }^{9}$. An epidemiological survey in France reported a lifetime prevalence of migraines and migrainous disorder of $20.3 \%{ }^{10}$. In contrast to this, a recent Norwegian study reported a lifetime prevalence of migraine in women of $34.1 \%{ }^{11}$.

Differences in reported prevalence may reflect a variety of factors across these studies. First, in the absence of consistent reporting of confidence intervals, it is unclear whether this difference is statistically relevant. In our own, smaller study, the confidence intervals of the reported prevalence encompass those of the previous Canadian studies, and are close to American prevalence rates.

If indeed there are true differences in prevalence, several factors could be considered, including survey methodology and calculation of either one-year or lifetime prevalence. One-year prevalence data, much like six-month prevalence data, such as those collected in the present study, may be less prone to recall bias than lifetime prevalence. Variation in reported prevalence may also be affected by other factors including modifications to the International Headache Society Classification criteria, cultural differences in symptom reporting, environmental factors across geographic regions (winds, hours of sunlight), and differences in health care systems and access ${ }^{12}$.

\section{Physician Consultation and Medication Use}

With the advent of new, effective, migraine-specific therapies over the last two decades, the study of medication utilization by migraine patients is warranted. It might be expected that medication use by women with migraine in Canada would have changed as new specific anti-migraine treatments became available. However, the likelihood of using these prescription medications is, by definition, dependent on whether migraine sufferers choose to see a prescribing physician about their headaches, and whether the diagnosis of migraine is made.

In this survey, nearly half of women with migraine never consulted a physician about their headaches. This number is slightly higher that reported by Edmeads et al for Canadian migraine sufferers in $1993^{13}$. It is also higher than that reported by Lipton et al for patients with migraine in the United States $(31 \%)$ and England $(14 \%)^{14}$. The reason for this difference between consulting practices is unclear. Bearing in mind that the 
present study is a small survey, another consideration is that some Canadian women choose to consult health professionals other than physicians, such as massage therapists, chiropractors, or others. The most important point about this finding is that nearly half of women suffering from disabling headaches do not consult their physicians about them.

With respect to medication use, our figures are similar to those in other studies. Fewer than $10 \%$ of Canadian women with migraine consider any specific anti-migraine medication (triptans or dihydroergotamine) to be their primary migraine treatment, whereas $27 \%$ primarily resort to opioids and other prescription medications, including butalbital. Similarly, in the United States and England, most people report using primarily over-the-counter medications to treat migraine headaches ${ }^{14}$. As in this Canadian survey, a survey for triptan use in four European countries and the United States found that only about half of the participants with migraine consulted a physician about their headaches, and of those only between $3 \%$ and $19 \%$ were prescribed triptans. As in our study, simple analgesics were the most commonly used treatments ${ }^{15}$.

There are two features of these data that are of particular importance. First, half of Canadian women have not seen a physician about their headaches, and only a small proportion of women are taking specific anti-migraine medications. This begs the question whether women with migraine are being undertreated for their condition, by virtue of not seeing a physician about their headaches.

While this is an important finding when looking at migraine management from a population standpoint, due consideration must also be given to the possibility that the number of individuals using specific anti-migraine medications is low because the population of women who have tried them previously may have found them to be ineffective or poorly tolerated.

The second important point to highlight from these data is the relatively frequent use of opioids and butalbital in the treatment of migraine. Standard first line treatments for migraine headaches are anti-inflammatories followed by triptans ${ }^{16}$. Opioids are recommended only for limited use because of the risk of developing medication-induced headache in the context of frequent migraine headaches ${ }^{16}$. Despite this, opioids and/or butalbital continue to be the primary medication for approximately $21 \%$ of Canadian women with migraine.

\section{Migraine Burden}

Migraine as a disease entity may represent two burdens to patients: the burden of psychosocial impact in addition to the pain and suffering associated with the headache itself. Migraine burden has been estimated in a variety of ways ranging from a socio-economic perspective to a psychological perspective.

For example, Pryse-Phillips et al estimated that seven million workdays are lost per year as a direct result of migraine headache in $\mathrm{Canada}^{4}$. In the United States, Hu et al reported 112 million bed-ridden days per year in the population of American migraine sufferers, with a cost to American employers of approximately $\$ 13$ billion dollars per year as a result of missed work and impaired work function ${ }^{17}$.

It has also been reported that migraine headache negatively impacts the emotional well-being of patients and families ${ }^{13}$. In a population based study, $79 \%$ of migraine sufferers reported that their headaches had an adverse effect on relationships and daily activities with family and friends were halted or bed-rest was required in $31 \%$ of reported migraine attacks. When responses were derived from actual headache diary data, social activities and family activities were cancelled in association with $17 \%$ of recorded migraine headaches ${ }^{13}$.

Lipton et al (2003) reported on the impact of migraine on family members and found that about one-third of partners of migraine-sufferers felt that arguments were more common because of headaches ${ }^{14}$.

In a telephone study about the emotional well-being of migraine sufferers in Europe, only $50 \%$ of patients had discussed their severe headaches with their physicians, and of these individuals, 39\% reported that they felt their physicians did not fully understand the extent to which their headaches interfered with their daily lives ${ }^{18}$. Further, $44 \%$ of patients reported feeling depressed because of their migraines ${ }^{18}$.

These figures are reflective of the fact that most migraine sufferers feel that their headaches have a substantial impact on their well-being. The findings from the Canadian Women and Migraine Survey suggest that the emotional impact of migraine headache for Canadian women is indeed very far-reaching, as approximately three quarters of women with migraine who participated in the survey reported feeling a lack of understanding or cynicism from those around them. Nearly all patients felt that they experienced a lack of control over their lives because of their migraines.

The psycho-social burden of migraine may be related in part to the psychological impact of missed work and daily activities that result from migraine headaches. The mean number of 'missed days' reported per year in this study was 20.8. In England, the mean number of missed work days per year reported in a population study was significantly lower, at 5.7 days $^{8}$. In another burden-of-disease study, Hu et al reported an average of 8.3 missed work days per year for women ${ }^{17}$.

The disparity in these values is likely due to the survey questions. "Missed days" in the Canadian survey was defined as days with reduced performance due to migraine AND days where work was missed. In addition, "work" was defined as work inside the home as well as employment outside the home.

$\mathrm{Hu}$ et al also took into account the number of days working with reduced function (7.6). If these two figures are summed, the number of 'missed days' identified in that study is 15.9 , which is quite in keeping with the findings of the present study ${ }^{17}$. The remaining disparity might be accounted for by the fact that $\mathrm{Hu}$ et al studied only working days (five days per week), whereas our survey did not distinguish between 'working days' or 'home days' (seven days per week). The other study only counted days missed from work or school, and did not include days missed from household duties, or non-work/non-school days ${ }^{8}$. It is interesting to note that Steiner et al calculated a correction of the 'missed days' to account for the severity of headache. When they removed the most severe headache sufferers (10\%) from the data set, the average number of missed days decreased to 0.9 days per year $^{8}$.

This finding bears out in the Canadian Women and Migraine survey as well; $20 \%$ of women with migraine missed no days, and nearly half missed five or fewer days. So, a majority of 
women missed less than the average number of days. In contrast, women who reported frequent migraines $(n=129)$ reported missing an average of 36.6 days per year. When these figures are taken into account on a population basis, the numbers are staggering. If $43 \%$ of the women in our sample reported frequent migraines, this translates to over 1.2 million women in Canada being partially or completely incapacitated by their migraines on average 36.6 days per year.

\section{Limitations}

There are several limitations of this study. The first is sample size. This study is small relative to some of the epidemiological studies that have been conducted previously. The second limitation is recall bias. Estimating period prevalence by patient recall is likely to underestimate prevalence. Using six-month prevalence as opposed to lifetime prevalence may mitigate this limitation to some extent. Finally, we acknowledge that the use of trained interviewers as opposed to formal clinical interviews by a specialist with expertise in headache disorders may affect the accuracy of headache diagnosis. While a clinical interview is the preferred method for making a headache diagnosis, for purposes of conducting a population-based telephone survey, using a standardized interview with trained interviewers is more feasible, and is a generally accepted method for conducting epidemiological research in this area.

\section{Conclusions}

In conclusion, the results of the Canadian Women and Migraine Survey indicate a prevalence of migraine in Canadian women of $26 \%$. As reported in other studies, many women with migraine headaches don't seek the help of a physician, and indeed, it may be that fewer Canadian women seek the help of a physician than in some other countries.

Medication use by Canadian women with migraine is still mainly comprised of over-the-counter medications followed by opioid and other prescription medications (NSAIDs, butalbital), whereas primary use of triptans and dihydroergotamine for treating migraines remains low, as seen in the United States and Europe.

Given that many women (over 400,000 ) are dissatisfied with their migraine treatments, most women with migraine feel isolated by the effects of suffering migraines, and relatively few women with migraine consult their physicians about the problem, it would appear that there is still a long way to go in identifying women with migraine headaches, and educating and supporting women in dealing with this common and debilitating condition. This is particularly important as the women with migraine in the Canadian Women and Migraine Survey reported a surprisingly high level of disability with at least partial incapacitation on average on almost 21 days a year in a population that represents three million Canadian women.

The findings of this study suggest that many women with migraine in our country do not have access to prescription medication for treating their migraines in part because they fail to see a prescribing physician about them. If we are to manage the enormous societal and individual burden of migraine in Canada, perhaps we must begin with improved public education: women with migraine need to understand that they have it, and that if their medication is insufficient to allow them to continue their daily activities, there may be other therapeutic options to help them.

\section{ACKNOWLEDGMENT}

This study was undertaken by Headache Network Canada with the support of a grant from Merck Frosst Canada.

\section{REFERENCES}

1. O'Brien B, Goeree R, Streiner D. Prevalence of migraine headache in Canada: a population-based survey. Int J Epidem. 1994;23(5): 1020-6.

2. Dowson AJ, Tepper SJ, Baos V, Baudet F, D'Amico D, Kilminster S. Identifying patients who require a change in their acute migraine treatment: The Migraine Assessment of Current Therapy (Migraine-ACT) questionnaire. Curr Med Res Opin. 2004;20:1125-5.

3. Headache Classification Committee of the International Headache Society. Classification and diagnostic criteria for headache disorders, cranial neuralgias, and facial pain. Cephalalgia. 1988; 8 Suppl 7:1-96.

4. Pryse-Phillips W, Findlay H, Tugwell P, Edmeads J, Murray TJ, Nelson RF. A Canadian population survey on the clinical, epidemiologic, and societal impact of migraine and tension-type headache. Can J Neurol Sci. 1992;19:333-9.

5. Stewart WF, Lipton RB, Celentano DD, Reed ML. Prevalence of migraine headache in the United States. Relation to age, income race, and other sociodemographic factors. JAMA. 1992;267:649.

6. Lipton RB, Stewart WF, Diamond S, Diamond ML, Reed M. Prevalence and burden of migraine in the United States: data from the American Migraine Study II. Headache. 2001;41: 646-57.

7. Lipton RB, Scher AI, Kolodner K, Liberman J, Steiner TJ, Stewart WF. Migraine in the United States: epidemiology and patterns of health care use. Neurology. 2002;58:885-94.

8. Steiner TJ, Scher AI, Stewart WF, Kolodner K, Liberman J, Lipton RB. The prevalence of adult migraine in England and its relationships to major sociodemographic characteristics. Cephalalgia. 2003;23(7):519-27.

9. Radtke A, Neurhauser H. Prevalence and burden of headache and migraine in Germany. Headache. 2009;49(1):79-89.

10. Henry P, Auray JP, Gaudin AF, Dartigues JF, Duru G, LanteriMinet M, et al. Prevalence and clinical characteristics of migraine in France. Neurology. 2002;59:232-7.

11. Russell MB, Kristiansen HA, Staltyte-Benth J, Kvaerner KJ. A cross-sectional population-based survey of migraine and headache in 21,177 Norwegians: the Akershus sleep apnea project. J Headache Pain. 2008;9:339-47.

12. Bigal ME, Lipton RB. The epidemiology, burden, and comorbidities of migraine. Neurol Clin. 2009;27:321-34.

13. Edmeads J, Findlay H, Tugwell P, Pryse-Phillips W, Nelson RF, Murray TJ. Impact of migraine and tension-type headache on lifestyle, consulting behaviour, and medication use: a Canadian population survey. Can J Neurol Sci. 1993;20:131-7.

14. Lipton RB, Bigal ME, Kolodner K, Stewart WF, Liberman JN, Steiner TJ. The family impact of migraine: population-based studies in the USA and UK. Cephalalgia. 2003;23(6):429-40.

15. MacGregor EA, Brandes J, Eikermann A. Migraine prevalence and treatment patterns: the Global Migraine and Zolmitriptan Evaluation Study. Headache. 2003;43:19-26.

16. Duncan CW, Watson DPB, Stein A. Diagnosis and management of headache in adults: summary of SIGN guideline. BMJ. 2008; 337:a2329-34.

17. Hu XH, Markson LE, Lipton RB, Stewart WF, Berger ML. Burden of migraine in the United States. Arch Intern Med. 1999;159; 813-18

18. Dueland AN, Leira R, Cabelli ST. The impact of migraine on psychological well-being of young women and their communication with physicians about migraine: a multinational study. Curr Med Res Opin. 2005;21(8):1297-305. 


\section{APPENDiX A \\ Canadian Women and Migraine Survey Questionnaire}

Q.1 Would you say you have had a headache often, occasionally, rarely or never in the last six months?

$\begin{array}{llll}\text { Often } & \text { Occasionally } & \text { Rarely } & \text { Never } \\ 1 & 2 & 3 & 4\end{array}$

If never, Skip to Q.10.

Q.2 When you have a bad headache, does it ever happen that...

Read in rotation Yes No

a)...the pain makes it difficult or impossible for you to get through your normal day

If NO, skip directly to Q.10

b)...your head hurts only on one side 1

c)...you feel throbbing pain (as though it were your pulse or a palpitation)

1

2

d)...the pain increases if you move 1

e)...you are sensitive to noise and/or light

f)...the headache is accompanied by nausea and/or vomiting

$$
12
$$

If YES to Q. 2a) AND YES to Q2b) or Q.2c) or Q.2d) AND YES to Q.2e) or Q.2f), check migraineur here

If not a migraineur, skip to Q.10

Q.3 Have you ever consulted a health care professional about your headaches?

$\begin{array}{ll}\text { YES } & 1 \\ \text { NO } & 2\end{array}$

Q.3 a) What type of health care professional did you consult? DO NOT READ

$\begin{array}{ll}\text { Doctor, physician } & 1 \\ \text { Nurse } & 2 \\ \text { Pharmacist } & 3 \\ \text { Ophthalmologist } & 4 \\ \text { Osteopath } & 5 \\ \text { Homeopath } & 6 \\ \text { Acupuncturist } & 7 \\ \text { Chiropractor } & 8 \\ \text { Other } & 9\end{array}$

Q.4 Which medication do you take (if many, ask for the principal one)? DO NOT READ

No medication taken Go to Q.8a)

Migraine drugs $\quad$ Other prescription drugs OTC (non-prescription)

Maxalt Antidepressant Advil (ibuprofen)

Amerge

Axert Celebrex

Imitrex
Anacin

Aspirin (ASA)

Motrin (ibuprofen)

$\begin{array}{lll}\text { Relpax } & \text { Excedrin } & \text { Tylenol (acetaminophen) } \\ \text { Zomig } & \text { Fiorinal } & \\ & \text { Migranal } & \\ & \text { Naproxen (Anaprox) } & \\ & \text { Percocet } & \\ & \text { Tylenol 2 } & \\ & \text { Tylenol 3 } & \text { Other: }\end{array}$

Q.5 Usually when you have a (migraine or bad headache)...

ROTATION Yes No

a)...are you able to function normally within two hours of taking your medication?

1

2

b)...does your headache disappear within two hours of taking your medication?

c)...does your medication work consistently, in the majority of your attacks?

1

2

d)....Are you comfortable enough with your medication to be able to plan your daily activities?

1

2

Q.6 Generally speaking, would you say that you are very satisfied, somewhat satisfied, a little unsatisfied or very unsatisfied...

with how effective your (the migraine or bad headache) medication is

$\begin{array}{ll}\text { Very satisfied } & 1 \\ \text { Somewhat satisfied } & 2 \\ \text { A little unsatisfied } & 3 \\ \text { Very unsatisfied } & 4\end{array}$

If somewhat satisfied or a little or very unsatisfied, ask Q.7a $+7 b$

Q.7a Have you ever asked your doctor if there are medications that would suit you better or that would be more effective?
YES
1
NO
2

Q.7b For what reason (ask this question whether participants answered yes or no)?

Q.8 a) How many days in the last six months would you estimate your (migraines/headaches) incapacitated you, including missed work days, difficulty doing housework and/or caring for children?

About days in the last 6 months

Q.8 b) How do you usually deal with your (migraine/bad headache) ? DO NOT READ (MULTIPLE ANSWERS ACCEPTED)

$\begin{array}{ll}\text { I take medication } & 1 \\ \text { Stay in bed until it's over } & 2 \\ \text { Stay in a dark room for a period of time } & 3 \\ \text { Protect myself against any noise } & 4 \\ \text { Other(specify) } & 5\end{array}$

1 c) How do you feel other people (family, friends, colleagues, employer) perceive the negative effects of migraine on your life? For example, missed work days or family time. Are they.... 
Totally sympathetic $\quad 1$

Somewhat sympathetic 2

Neutral 3

Somewhat unsympathetic 4

Totally unsympathetic 5

Q.9 Do you totally agree, somewhat agree, somewhat disagree or totally disagree that the following statements describe your situation (condition) well?

Rotation

$\begin{array}{lr}\text { Totally agree } & 1 \\ \text { Somewhat agree } & 2 \\ \text { Somewhat disagree } & 3 \\ \text { Totally disagree } & 4 \\ \text { D.N.K } & 5\end{array}$

People who suffer from (migraines or bad headaches) sometimes feel a lack of control over their lives

$\begin{array}{lllll}1 & 2 & 3 & 4 & 5\end{array}$

(Migraines or bad headaches) can keep sufferers from attending work, school and/or family activities

$\begin{array}{lllll}1 & 2 & 3 & 4 & 5\end{array}$

People who suffer from (migraines or bad headaches) are misunderstood, or not taken seriously, when it comes to their condition 1

2

3

4

5

Q.10 How old are you?

$\begin{array}{ll}18-24 & 1 \\ 25-34 & 2 \\ 35-44 & 3 \\ 45-54 & 4 \\ 55-64 & 5 \\ 65 \text { and over } & 6\end{array}$

Province

2
3
4
5
6

